Ann. Zootech., I977, 26 (3), 355-377.

\title{
Déterminisme psychophysiologique de l'entrée des vaches en salle de traite
}

\author{
Nicole ROSE et J. LABUSSI ÈRE \\ avec la collaboration technique de F. A. de la Chevalerie \\ Laboratoive de la Traite, \\ Centre de Recherches de Rennes, I.N.R.A., \\ 65 , rue de Saint-Brieuc \\ 35042 Rennes Cedex (France)
}

\section{Résumé}

L'ordre et le choix d'entrée de 57 vaches FFPN dans 2 salles de traite " tandem " et dans les 2 parcs d'attente (grillagé et cimenté) qui y sont adjoints (fig. I) ont été contrôlés 60 fois lors de la traite du matin.

Il ressort de cette étude que :

$\mathrm{I}^{\mathrm{o}} 4$ vaches sur 5 environ $(80,7$ p. I00) choisissent presque toujours la même aire d'attente puisque $26,3 \mathrm{p}$. Ioo et $54,4 \mathrm{p}$. Ioo du troupeau optent respectivement pour le parc grillagé et cimenté dans plus de 90 p. roo des cas (tabl. I).

$2^{\circ}$ L'ordre d'entrée en salle de traite est régulier mais l'accès en salle d'attente est plus variable (tabl. 2 et fig. 2).

L'analyse des correspondances (fig. 3) entre l'ordre d'entrée des animaux et 22 paramètres permettant de caractériser les vaches (tabl. I) indique que :

I $^{0}$ Un groupe de bonnes laitières manifeste l'ordre d'entrée le plus stable et la fréquence cardiaque la plus basse. Le coefficient de corrélation entre la production maximum en première lactation et la fréquence cardiaque est alors de - o,5I.

$2^{\circ}$ Un groupe de bonnes laitières rentre en tête en salle de traite (et inversement).

$3^{\circ}$ Il n'y a pas de relation entre l'hématocrite et l'ordre d'entrée en salle de traite.

$4^{0}$ Celui-ci n'est également relié ni à l'âge ni au poids de l'animal.

$5^{\circ}$ L'âge et le poids par contre influencent le rang hiérarchique puisque les vaches dominantes sont souvent les plus âgées, les plus lourdes et les plus anciennes dans le troupeau (et inversement).

$6^{\circ}$ L'ordre d'entrée en salle de traite ne semble pas dépendre de la place dans la hiérarchie mais certaines vaches dominantes pourraient se servir de leur "supériorité " pour régler à leur profit certains conflits et gagner ainsi quelques places si elles le désirent.

Une étude complémentaire à l'analyse des correspondances indique que les vaches qui pénètrent les premières en salle de traite sont également celles pour lesquelles l'apparition du réfexe d'éjection du lait est la plus fréquente lors de cette entrée et surtout lors de la distribution d'aliment au poste de traite (les corrélations entre ces 2 stimulants de l'éjection et le rang d'entrée sont respectivement de $-0,47$ et $-0,80$, (tabl. 4).

L'apport de concentré en salle de traite faciliterait donc l'accès des animaux dans celle-ci et la suppression de la ration retarde l'ordre d'entrée de 80 p. Ioo des animaux soumis à cette privation. On peut supposer que les stimuli qui précèdent l'éjection du lait tels que le stationnement dans le parc d'attente, l'ouverture et le passage de la porte d'entrée en salle de traite, l'installation dans une stalle, le bruit des pulsateurs, l'arrivée du vacher, la récompense alimentaire 
sont susceptibles de devenir des signaux dont l'enchaînement très complexe serait continuellement renforcé par l'excitation mécanique des trayons. Dans ce contexte, les sujets de type " fort équilibré mobile " de la terminologie Pavlovienne pourraient être ceux qui rentrent les premiers en salle de traite.

\section{Introduction}

La rapidité de traite d'un troupeau dépend pour une grande part de la vitesse avec laquelle les vaches accèdent en salle de traite (LABussière, 1975).

Il est bien connu qu'à l'approche de l'heure de la traite, les animaux se rassemblent eux-mêmes à proximité du parc d'attente et les éleveurs savent bien que si leur entrée en salle de traite s'effectue chaque jour selon un ordre presque identique, il faut perdre parfois beaucoup de temps pour aller pousser certains sujets réticents.

Cette connaissance empirique a été confirmée par quclques travaux de recherche.

S'il semble admis que cette régularité se maintient d'une lactation à l'autre (Willems et LAMPO, I964) et est particulièrement nette pour les vaches qui rentrent en tête et en queue (WiLlems et Lampo, I964 - Hidari, Kido et Suzu ki, I973), les avis sont par contre beaucoup plus partagés pour en déterminer les causes. seraient :

En effet, les animaux qui ont tendance à accéder les premiers en salle de traite

a) Les meilleures laitières pour Willems et Lampo (I964), Fergusson et al. (I967), Kostov et Subev (I970) mais Dietrich (I962), Dietrich et al. (I965), HidARI, Kido et SUzu KI (I973), REINHARDT (I973) ne trouvent à cet égard aucun effet du niveau de production.

b) Les plus vieux pour Willems et Lampo (I964), Fergusson et al. (I967), Kostov et Subev (I970) (vaches de 5 à 6 ans) mais Dietrich (I962), Dietrich et al. (I965), Hidari, Kido et Suzu Ki (I973), Reinhardt (I973) n'ont relevé aucune incidence de l'âge (ni du poids) sur l'ordre d'entrée.

DieTrich (I962) et DiCKSON, BARR et Wieckert (I967) estiment enfin que la place dans la hiérarchie sociale n'affecte pas le rang de passage mais là encore ce point de vue n'est partagé ni par Guhl et Atkeson (I959) ni par Reinhardt (I973).

Les contradictions relevées à la lecture de ces quelques résultats peuvent certainement trouver leur justification dans les différences rencontrées en ce qui concerne la taille des troupeaux, leur race, la durée des observations et l'utilisation des méthodes statistiques qui ne permettent pas une compréhension suffisante des données recueillies.

C'est pourquoi il nous a paru justifié d'essayer d'apporter de nouveaux éléments pour éclaircir cette question, en utilisant un nombre de vaches important et une méthode d'interprétation plus appropriée à ce type de problème. Une meilleure compréhension du déterminisme psychophysiologique de l'entrée des vaches en salle de traite permettrait en effet très probablement d'améliorer l'organisation du travail du vacher surtout si l'on sait que les systèmes de chiens mécaniques ou de chiens électriques sont généralement peu satisfaisants à cause de leur prix ou de leurs dangers (perturbation, voire inhibition du réflexe d'éjection du lait). 


\section{II. - Matériel et méthodes}

\section{A. - Description des locaux expérimentaux}

I. La stabulation libre.

La stabulation de $30 \mathrm{~m} \times \mathrm{I} 7 \mathrm{~m}$ (soit environ II $\mathrm{m}^{2}$ par vache) est décrite à la figure I $\left(^{1}\right)$. Chaque animal a librement accès à une surface paillée couverte et à une aire cimentée découverte. Les façades nord, est et ouest sont " bardées » par un mur qui assure une protection contre les intempéries et les vents dominants.

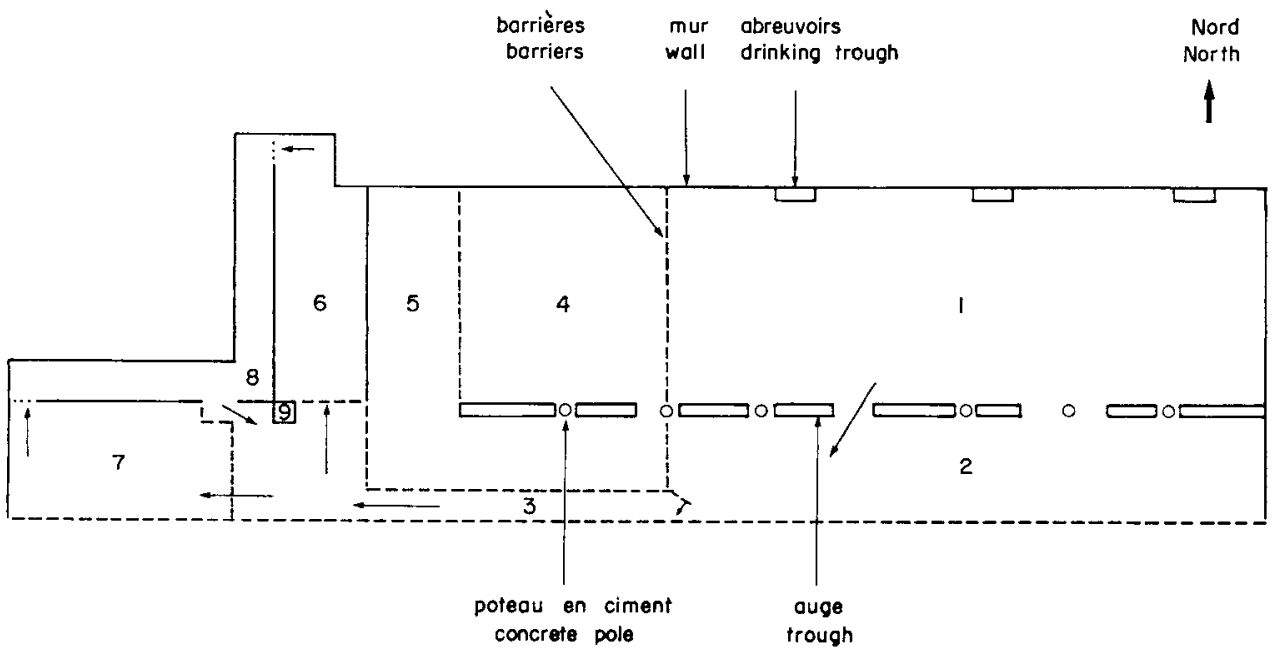

FIG. I. - Plan du bâtiment où sont effectuées les observations. Building where the observations were made.

I. Stabulation libre (aire paillée) (loose housing (straw litter)).

2. Stabulation libre (aire cimentée) (loose housing (concrete floor)).

3. Couloir (general passage).

4. Parc réservé aux vaches taries (pen for $d r y$ cows).

5. Parc réservé aux expériences (pen for experiments).

6. Parc d'attente grillagé (waiting-pen with slatted floor).

7. Parc d'attente cimenté (waiting-pen with concrete floor).

8. Salle de traite (milking-parlour).

9. Cabine d'observation (observation cabin).

La partie sud est fermée par des barrières délimitant un couloir de $\mathrm{I}, \mathrm{I} 5 \mathrm{~m}$. de large permettant l'accès aux parcs d'attente de la salle de traite.

\section{Parcs d'attente et salle de traite.}

A la sortie du couloir venant de la stabulation, chaque vache a le choix entre 2 parcs d'attente : celui de gauche, au sol cimenté est ouvert au sud (barrières de I,35 m de hauteur) et fermé à l'ouest; celui de droite, au sol grillagé est plus sombre (entouré de 3 murs) et il importe de remarquer que la grille du sol surplombant

(1) Ces normes sont proches de celles préconisées par le Ministère de l'Agriculture : $\mathrm{I}_{3} \mathrm{~m}^{2}$ par vache laitière. (L'étable à stabulation libre avec ou sans paille. Imprimerie nationale de Paris, I965.) 
TABLEAU I

Caractéristiques des 57 vaches FFPN du troupeau Characteristics of the 57 FFPN cows in the herd

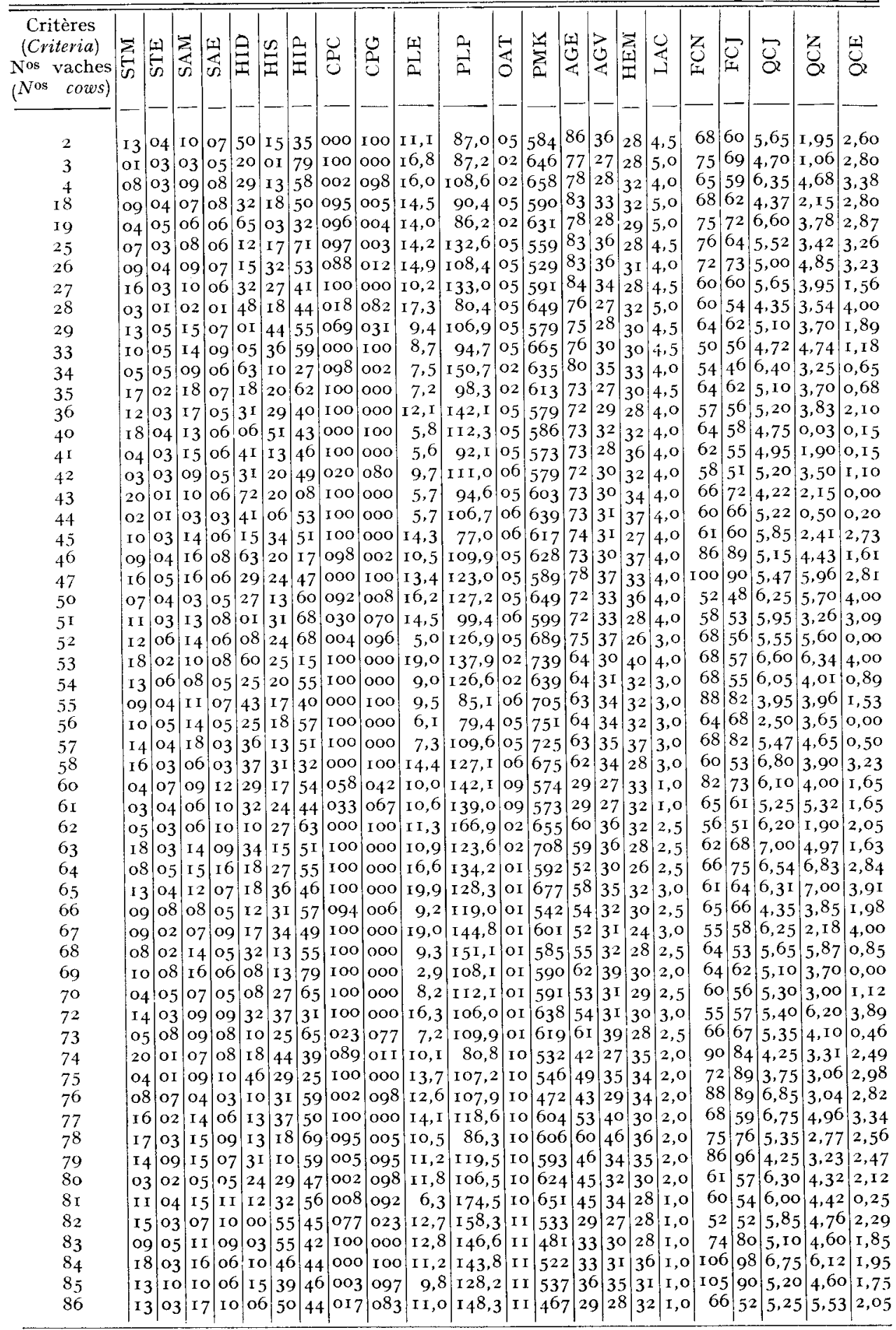


une fosse à lisier il peut en résulter une impression de "vide " susceptible d'in fluencer le choix de certains animaux. Chaque aire d'attente donne accès à 4 stalles "tandem " de la salle de traite par une porte coulissante d'une largeur d'i mètre; l'ouverture et la fermeture de cette porte sont commandées de l'intérieur par l'ouvrier trayeur grâce à un système de câbles guidés par des poulies. Les vaches ont à franchir 3 marches avant d'entrer en salle de traite (dénivellation totale de $50 \mathrm{~cm}$ environ). La sortie, suivie de quelques marches, est commune aux 8 stalles. La cabine où nous avons effectué nos observations est placée à $\mathrm{r}, 50 \mathrm{~m}$ au-dessus du sol au carrefour des voies de passage des animaux entrant dans les parcs et sortant de la salle de traite.

Caractéristiques des 57 vaches IFFN du troupeau

Characteristics of the 57 FFPN cotes in the herd

STM : médiane de l'ordre d'entrée en salle de traite. median of the order of entrance into the milking parlour.

STE : écart de l'ordre d'entrée en salle de traite. deviation of the order of entrance into the milking parlour.

SAM : médiane de l'ordre d'entrée en salle d'attente. median of the order of entrance into the waiting-pen.

SAE : écart de I'ordre d'entrée en salle d'attente. deviation of the order of entrance into the waiting-pen.

HID : index de dominance (p. 100). dominance index (p. Ioo).

HIS : index de subordination (p. Ioo). subordination index (p. Ioo).

HIP : index de passivité (p. Ioo). passivity index (p. I oo).

CPC : choix du parc cimenté (p. Ioo). choice of pen with concrete floor (p, 10o).

CPG : choix du parc grillagé (p. Ioo). choice of pen with slatted floor (p. roo).

PLE : production laitière au cours de l'expérience (I). milk production during the experiment $(l)$.

PLP : production laitière maximum en premièrc lactation (1) maximum milk production in Ist lactation $(l)$.

OAT : no d'ordre d'arrivée dans le troupeau. order of arrival in the herd.

PMK : poids moyen $(\mathrm{kg})$. average weight $(\mathrm{kg})$.

$\mathrm{AGE}$ : âge (mois). age (months).

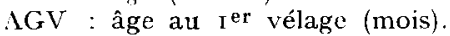
age at Ist calving (months).

HEM : hématocrite. hematocrit.

LAC : numéro de lactation. lactation order.

FCN : fréquence cardiaque après $5 \mathrm{~h}$ de jeûne. cardiac frequency after $5 \mathrm{hrs}$ of fasting.

FC J : fréquence cardiaque après 24 h de jeûne. cardiac frequency after $2+$ hrs of fasting.

QCJ : quantité de concentré ingérée après $24 \mathrm{~h}$ de jeûne $(\mathrm{kg})$. concentrate intake after 27 hrs of fasting $(\mathrm{kg})$.

QCN : quantité de concentré ingérée après $5 \mathrm{~h}$ de jê̂ne (kg). concentrate intake after 5 hrs of fasting $(k g)$.

QCE : quantité de concentré ingérée au cours de l'expérience (kg). concentrate intake during the experiment $(\mathrm{kg})$. 


\section{B. - Caractéristiques du troupeau et méthodes d'élevage}

Le troupeau se compose de 57 vaches Française Frisonne Pic Noire qui proviennent :

- du transfert à Rennes en I97I d'un " noyau " expérimental de 35 têtes déjà constitué dans une autre station (Jouy-en-Josas),

- de l'achat à partir de cette date de 22 génisses prêtes à vêler qui ont été intégrées (au fur et à mesure de leur arrivée) au groupe précédent. Un numéro d'ordre d'arrivée dans le troupeau (OAT) est attribué arbitrairement à chaque animal en fonction de sa date d'intégration dans celui-ci.

Les traites ont lieu à 7 h et à $\mathbf{I} 6$ h 30 . Un ouvrier va chercher les vaches « en lait " dans la stabulation; il les dirige calmement de la voix ou parfois en les poussant avec un bâton vers le couloir à la sortie duquel les vaches s'engagent librement dans les aires d'attente cimentées et grillagées où elles vont être enfermées. Lorsque les 2 portes de la salle de traite s'ouvrent, une première série de 4 animaux par parc rentre simultanément de chaque côté. Chacun des animaux se place dans une stalle et reçoit une ration d'aliment concentré calculée en fonction de sa production laitière. La pose des gobelets est immédiatement précédée d'un nettoyage de la mamelle avec un linge trempé dans un seau d'eau chaude à $45^{\circ}$. La dépose fait suite à un égouttage qui commence lorsque le débit du lait devient inférieur à $200 \mathrm{~g}$ par minute.

Chaque laitière libère alors sa stalle et regagne la stabulation; la porte s'ouvre à nouveau sur le parc d'attente et l'animal suivant rentre de lui-même ou bien est sollicité par le vacher.

Les 22 paramètres que nous avons retenus pour caractériser chaque animal et qui seront traités par analyse des correspondances sont présentés au tableau r. Le mode d'obtention et d'expression de certains d'entre eux est explicité au paragraphe ci-dessous.

\section{C. - Méthode expérimentale et mode d'expression des résultats}

\section{Description du choix du parc d'attente et de l'ordre d'entrie.}

L'entrée dans les aires d'attente et dans la salle de traite a été contrôlée 60 fois lors de la traite du matin entre le 20 février I973 et le 21 juin I973. Depuis la cabine d'observation, nous notons pour chaque vache :

- leur choix pour le parc d'attente cimenté (CPC) ou grillagé (CPG) rapporté dans les 2 cas en pourcentage du nombre total d'essais,

- leur rang d'entrée en salle d'attente puis en salle de traite.

Étant donné que le nombre total de vaches entrant dans chaque parc est variable $\left({ }^{1}\right)$, la valeur directe du rang d'entrée n'est pas comparable d'un contrôle à l'autre $\left({ }^{2}\right)$. Pour pallier cet inconvénient et exprimer l'ordre d'entrée en fonction du nombre total de sujets dans le parc d'attente, on ramène arbitrairement ce nombre à IOO.

(1) Ceci est dû aux changements d'effectifs (tarissement, vêlage) mais aussi au fait que l'animal peut modifier son choix et changer de parc d'attente. sur 35 .

(2) On ne peut en effet, accorder la même signification au fait qu'une vache soit r 8 e sur 25 ou 18 e 


\section{Détermination du rang social.}

a) Sur le troupeau entier (HID, HIS, HIl).

Les observations concernant la hiérarchie du troupeau sont effectuées d'une part, à la traite du matin lors de la présence des animaux dans les parcs d'attente et d'autre part, vers i $h$ lors de la distribution des aliments en stabulation. C'est ce dernier contrôle qui apporte le maximum de renseignements en raison du regroupement de tous les animaux $\left(^{1}\right)$ et de l'intensité des rapports exacerbés par la compétition alimentaire.

Plusieurs formes de rapport entre les vaches permettent de déterminer leur hiérarchie sociale (Boussou, I964). En ce qui nous concerne, nous nous sommes limités à considérer comme dominant l'animal qui donne des coups de tête (avec contact) sans en recevoir.

Ce type d'appréciation permet de définir :

- l'Index de dominance (HII))

- l'Index de subordination (HIS)

qui expriment respectivement la proportion de sujets qu'une vache domine, ou qui la domine, lorsque l'effectif du troupeau est ramené à Ioo,

l'Index de passivité (HIP) qui représente le complément à Ioo de la somme des 2 index précédents. Il caractérise une absence de contacts sociaux due :

- soit à l'insuffisance d'observations $\left({ }^{2}\right)$,

- soit au comportement effectivement passif de l'animal qui évite les rencontres avec ses congénères,

- soit au choix du critère retenu (coups de tête) qui néglige certaines formes d'expression des relations hiérarchiques telles que les postures de tête définies par Bouissou en ig64.

b) Sur un groupe de I2 vaches.

Étant donné qu'il est difficile d'établir en totalité la hiérarchie d'un troupeau d'une soixantaine de vaches, nous avons cherché à déterminer le rang hiérarchique avec plus de précision en nous limitant à I 2 animaux. La méthode utilisée est celle de la compétition alimentaire décrite par Botissol, en I970.

Les sujcts sont introduits par paire (soit 66 combinaisons) dans un parc où l'on a disfosé un seau rempli d'aliment concentré. La vache dominante mange seule pendant la totalité du test; si la vache dominée essaie d'accéder au seau quand la dominante lève la tête, elle est immédiatement évincée par une menace ou un coup.

Afin d'établir le rang social de chaque animal, on utilise le " pouvoir " qui représente le nombre d'animaux qu'il domine et le (pouvoir) 2 qui est la somme de son propre pouvoir et de celui des vaches qui lui sont hiérarchiquement inférieures.

\section{Détermination de la fréquence cardiaque ( $F C J$ et $F C N)$.}

Celle-ci est appréciée au moyen d'un stéthoscope après un jêne de $20 \mathrm{~h}$ (FCJ) et de $5 \mathrm{~h}(\mathrm{FCN})$. L'examen a lieu à $\mathrm{I}_{4} \mathrm{~h}$ dans le parc d'attente cimenté où les vaches ont été mises préalablement au repos pendant $15 \mathrm{mn}$.

(1) Les vaches se répartissant dans les 2 pares d'attente, on ne retrouve dans chacun d'entre eux qu'une partie des animaux et ainsi toutes les intéractions ne peuvent se manifester.

(') Nous n'avons pu observer les intéractions qu'entre 875 couples sur les I 596 paires potentielles du troupeau. 


\section{Détermination de l'hématocrite (HEM).}

Le sang est prélevé à la veine jugulaire dans un tube contenant un anticoagulant (Héparine) puis transféré dans un délai maximum de 2 à $3 \mathrm{~h}$ dans 2 microtubes pour chaque vache; l'une des extrémités est fermée à la flamme et les microtubes sont centrifugés pendant $2 \mathrm{mn}$ à la vitesse de I 800 tours par minute. La valeur des éléments figurés du sang est lue directement sur une échelle graduée de o à roo.

\section{Rôle de l'aliment concentré ( $Q C E, Q C N, Q C J)$.}

Chaque vache est caractérisée à cet égard par :

a) La quantité moyenne d'aliment qu'elle reçoit chaque matin en salle de traite au cours de l'expérience (QCE) mais aussi par la quantité maximum qu'elle est capable d'ingérer pendant un test de ro mn après un jeûne de $20 \mathrm{~h}(\mathrm{QC} J)$ et de $5 \mathrm{~h}(\mathrm{QCN})$.

b) L'apparition du réflexe d'éjection du lait qui peut éventuellement être provoqué par la distribution de la ration. Ce réflexe est détecté par :

- l'écoulement du lait alvéolaire à travers une canule placée dans le trayon,

- l'accroissement de la pression intramammaire enregistrée selon la technique décrite par LABUSSIÈRE et DLRAND (I970).

En outre, au cours d'un essai complémentaire sur io animaux, la ration quotidienne de concentré a été supprimée aux 5 sujets entrant en tête en salle de traite et au contraire augmentée (jusqu'à $6 \mathrm{~kg}$ ) pour les 5 derniers.

\section{Influence du degré de réplétion de la mamelle (PLE).}

Le degré de réplétion de la mamelle pouvant inciter certaines vaches à venir se faire traire plus rapidement, nous avons cherché à vérifier ce point en contrôlant la quantité de lait présente dans la glande lors de l'entrée en salle de traite (PLE).

\section{III. - Résultats}

\section{A. - Répétabilité de l'entrée en salle d'attente et en salle de traite}

\section{Choix du parc d'attente.}

Un récapitulatif des valcurs rapportées aux colonnes CPC et CPG du tableau I indique que 4 vaches sur 5 environ $(80,7 \mathrm{p}$. IOO) choisissent toujours la même aire d'attente puisque :

des cas, 3 I $(54,4$ p. Ioo du troupeau) optent pour le parc cimenté dans go à Ioo p. Ioo des cas,

- I r seulement (I9,3 p. Ioo du troupeau) n'ont pas de préférence aussi nette (CPC et CPG inférieurs à 90 p. IOO).

\section{Ordre d'entrée en salle d'atiente et en salle de traite.}

A titre d'exemple, nous présentons au tableau 2 les rangs d'entrée de 4 vaches. On remarquera que la vache $n^{\circ} 44$ occupe toujours les premières places alors que la vache $n^{0} 43$ a tendance à accéder régulièrement parmi les dernières en salle de traite et on notera qu'il n'y a pas concordance stricte entre le rang d'entrée en salle d'attente et en salle de traite. 
TABLEAU 2

Exemple de l'ordve d'entrée en salle de traite et en salle d'attente de 4 vaches du troupeau Order of entrance into the milking parlour and into the waiting-pen of 4 corss in the herd

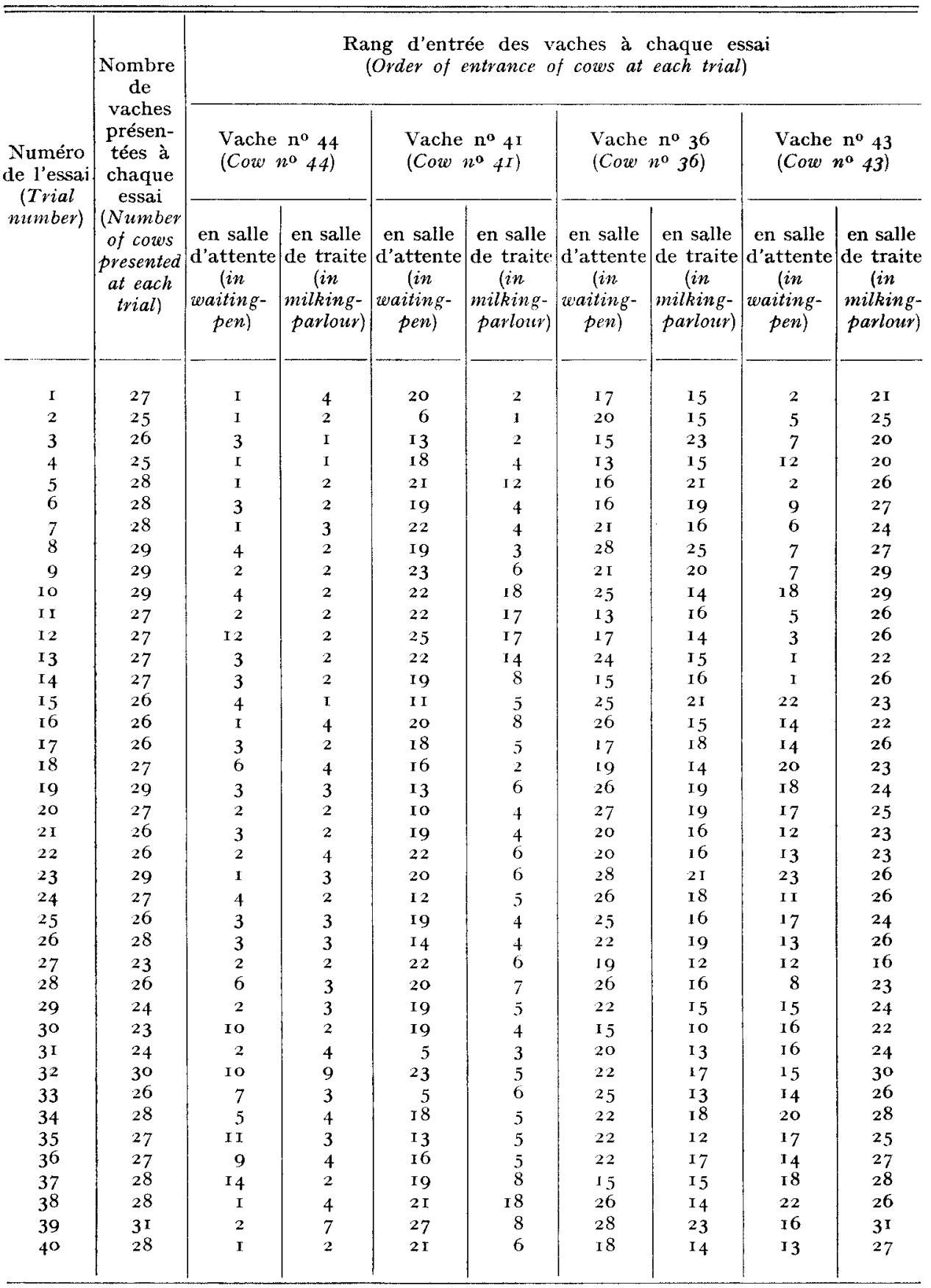


TABLEAL 2 - TABLE 2 (suite)

\begin{tabular}{|c|c|c|c|c|c|c|c|c|c|}
\hline \multirow{3}{*}{$\begin{array}{l}\text { Numéro } \\
\text { de l'essaj } \\
\text { (Trial } \\
\text { numbet })\end{array}$} & \multirow{3}{*}{$\begin{array}{c}\text { Nombre } \\
\text { de } \\
\text { vaches } \\
\text { présen- } \\
\text { tées à } \\
\text { chaque } \\
\text { essai } \\
\text { (Number } \\
\text { of cows } \\
\text { presented } \\
\text { at cach } \\
\text { trial) }\end{array}$} & \multicolumn{8}{|c|}{$\begin{array}{l}\text { Rang d'entrée des vaches à chaque essai } \\
\text { (Order of entrance of cows at each trial) }\end{array}$} \\
\hline & & \multicolumn{2}{|c|}{$\begin{array}{l}\text { Vache } n^{\circ}+4 \\
\left(\text { Cow } n^{\circ}+4\right)\end{array}$} & \multicolumn{2}{|c|}{$\begin{array}{l}\text { Vache } n^{0}+1 \\
\left(\text { Cow } n^{0}+{ }^{I}\right)\end{array}$} & \multicolumn{2}{|c|}{$\begin{array}{l}\text { Vache } n^{\circ} 3^{6} \\
\left(\text { Cow } n^{\circ} 3^{6}\right)\end{array}$} & \multicolumn{2}{|c|}{$\begin{array}{l}\text { Vache } n^{0}+3 \\
\left(\text { Cow } n^{0}+3\right)\end{array}$} \\
\hline & & $\left\{\begin{array}{c}\text { en salle } \\
\text { d'attente } \\
\text { (in } \\
\text { waiting- } \\
\text { pew) }\end{array}\right.$ & $\begin{array}{c}\text { en salle } \\
\text { de traite } \\
\text { (in } \\
\text { milking } \\
\text { parlour) }\end{array}$ & $\begin{array}{c}\text { en salle } \\
\text { d'attente } \\
(\text { in } \\
\text { waiting- } \\
\text { pen })\end{array}$ & $\begin{array}{c}\text { en salle } \\
\text { de traite } \\
\text { (in } \\
\text { milking- } \\
\text { parlour) }\end{array}$ & $\begin{array}{c}\text { en salle } \\
\text { d'attente } \\
\text { (in } \\
\text { waiting- } \\
\text { pen) }\end{array}$ & $\begin{array}{c}\text { en salle } \\
\text { de traite } \\
\text { (in } \\
\text { milking- } \\
\text { parlouv) }\end{array}$ & $\begin{array}{c}\text { en salle } \\
\text { d'attente } \\
\text { (in } \\
\text { waiting- } \\
\text { pen) }\end{array}$ & $\begin{array}{c}\text { en salle } \\
\text { de traite } \\
\text { (in } \\
\text { milking- } \\
\text { parloutr) }\end{array}$ \\
\hline+1 & 33 & 2 & 3 & 17 & 5 & 15 & $\mathrm{I}_{4}$ & 9 & 30 \\
\hline 42 & 30 & 2 & 2 & I 5 & 6 & 2.5 & I 8 & 16 & 29 \\
\hline 43 & $3 \mathrm{I}$ & 5 & 6 & 22 & 10 & 30 & I 8 & $I_{5}$ & 30 \\
\hline $4+$ & $3 I$ & I & 2 & 24 & 8 & 26 & I 5 & 15 & 31 \\
\hline 45 & 29 & I I & 4 & 19 & 6 & 23 & 18 & 18 & 27 \\
\hline 46 & 29 & I & 2 & 20 & 8 & 26 & I 2 & 10 & 29 \\
\hline 47 & 30 & 4 & 4 & 23 & 3 & 28 & $I_{4}$ & 18 & 29 \\
\hline $4^{8}$ & $3^{2}$ & I & 3 & 28 & 10 & 27 & I 4 & I3 & 29 \\
\hline 49 & $3 \mathrm{I}$ & 7 & 2 & 19 & 4 & 31 & I 4 & I 7 & 30 \\
\hline 50 & 33 & 7 & 3 & I6 & 2 & 23 & 20 & 15 & $3^{1}$ \\
\hline .51 & 36 & I & 5 & 7 & I & $3^{2}$ & 25 & I 8 & $3^{6}$ \\
\hline 52 & 36 & $\mathrm{I} 2$ & 2 & 30 & 7 & 31 & 22 & 19 & $3^{6}$ \\
\hline .53 & 3.5 & 6 & 6 & 26 & 5 & 34 & 20 & 19 & 33 \\
\hline .54 & 36 & 16 & 3 & $2 \mathrm{I}$ & 6 & 32 & 24 & I 7 & 35 \\
\hline 55 & 34 & .5 & 2 & 29 & 5 & 33 & 19 & 13 & $3+$ \\
\hline 56 & 34 & 6 & 2 & 29 & 5 & 34 & 21 & $2 \mathrm{I}$ & $3+$ \\
\hline .57 & 36 & 2 & 3 & 27 & + & 26 & 23 & 17 & $3^{6}$ \\
\hline .58 & $3^{6}$ & II & 7 & 26 & 9 & 33 & 20 & 24 & 35 \\
\hline 59 & 34 & 8 & + & 13 & 6 & 33 & 29 & 19 & 33 \\
\hline 60 & 37 & 5 & 5 & 27 & 9 & 36 & $3 I$ & 28 & 37 \\
\hline
\end{tabular}

L’ensemble des résultats obtenus pour les 57 animaux est rassemblé à la figure 2. L'histogramme des ordres d'entrée médian en salle de traite (STM) montre qu'il y a toujours environ 3 animaux par classe (ce qui correspond à la répartition moyenne de 57 vaches dans 20 classes) et ceci laisse supposer que les vaches conservent toujours approximativement le même rang d'entrée en salle de traite tout au long de l'essai ( $\left.{ }^{1}\right)$. Cette régularité est confirmée par les faibles valeurs des quartiles (STE) qui restent inférieurs ou égaux à 5 pour 85 p. Ioo du troupeau.

Par contre, la répétabilité de l'ordre d'entrée en salle d'attente semble beaucoup moins bonne. Les médianes (SAM) se répartissent d'une façon moins régulière dans chacune des 20 classes et surtout les quartiles (SAE) sont beaucoup plus élevés que dans le cas précédent ( $85 \mathrm{p}$. I 00 du troupeau entre les valeurs 5 et Io).

Ceci est peut-être dù au fait que les vaches sont alors perturbées par l'ouvrier

(1) İn effet, si l'ordre d'entrée avait été aléatoire pour les 57 vaches au cours de tous les contrôles nous aurions eu un grand nombre d'animaux à médiane $r o$. 
qui est chargé de les pousser dans l'étroit couloir d'accès aux parcs d'attente alors qu'elles disposent ensuite de beaucoup plus de temps pour déterminer librement leur entrée en salle de traite.

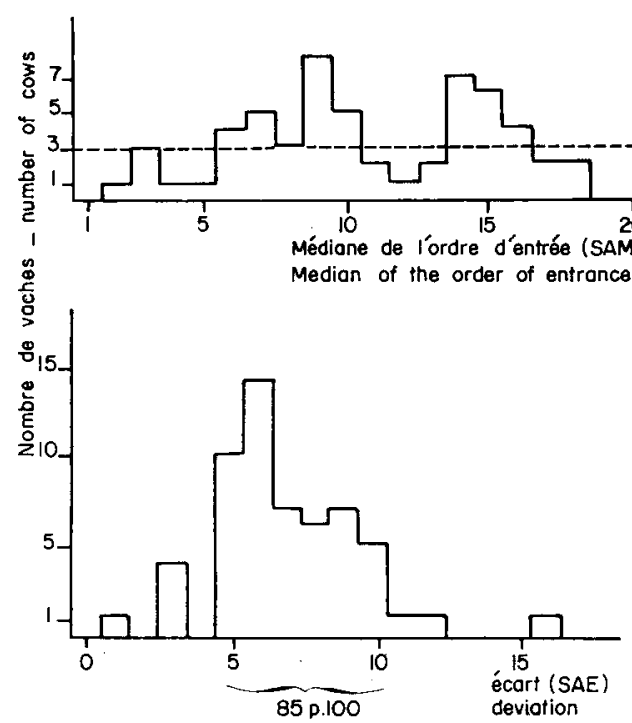

du troupeau - of herd
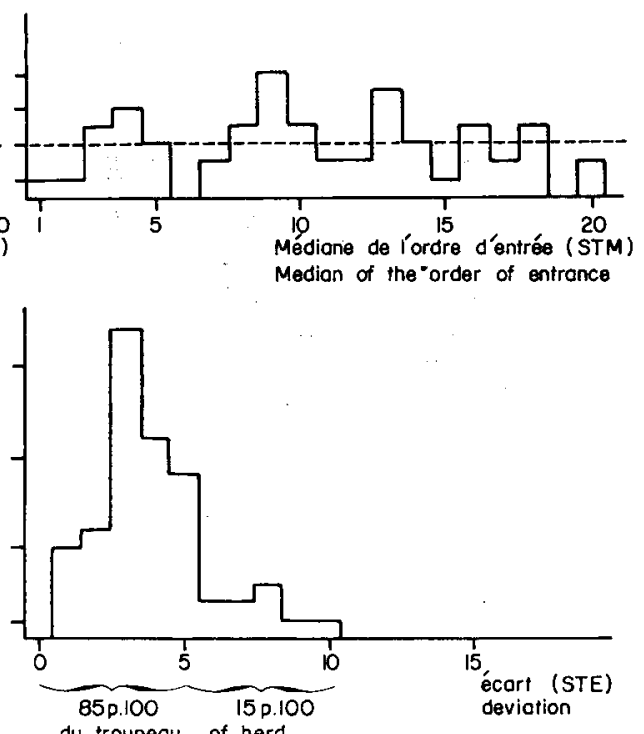

du troupeau - of herd

FIG. 2.- Histogramme des ordres d'entrées en salle d'attente et en salle de traite exprimés par leur médiane et leur quartile.

Histogram of orders of entrance into the waiting-pen and milking-parlour $b y$ their median and their quartile.

2a. Histogramme des ordres d'entrée en salle d'attente (médiane SAM) (histogram of orders of entrance into the waiting-pen ( $S A M$ median).

2b. Histogramme des écarts (quartiles) des ordres d'entrée en salle d'attente (SAE) (histogram of deviations (quartiles) of orders of entrance into the waiting-pen (SAE).

2c. Histogramme des ordres d'entrée en salle de traite (médiane STM) (histogram of orders of entrance into the milking-parlour (STM median).

$2 d$. Histogramme des écarts (quartiles) des ordres d'entrée en salle de traite (histogram of deviations (quartiles) of orders of entrance into the milking-parlonr).

\section{B. - Analyse des correspondances entre les parametres pouvant influencer l'entrée en salle d'attente}

Vingt parmi les vingt-deux ( ${ }^{1}$ ) paramètres caractérisant les 57 vaches du troupeau sont traités à l'ordinateur selon un programme d'analyse factorielle des correspondances. Les résultats sont présentés à la figure 3 sur un système de 4 plans définis par 5 axes qui contiennent respectivement 38, I p. IO0, I8,8 p. IO0, Ir, 4 p. IOO, 9, I p. Ioo et 5,6 p. Ioo de l'information statistique totale. Rappelons que chaque vache ou chaque paramètre est représenté dans le plan par un point défini

(1) Pour des raisons que nous expliquerons au paragraphe 5 , nous avons dú supprimer les 2 paramètres $\mathrm{CPC}$ et $\mathrm{CPG}$. 
sur l'axe où il se projette non seulement par ses coordonnées mais aussi par un coefficient de contribution relative (voir tabl. 3) qui précise l'importance qu'il faut accorder à cette projection.
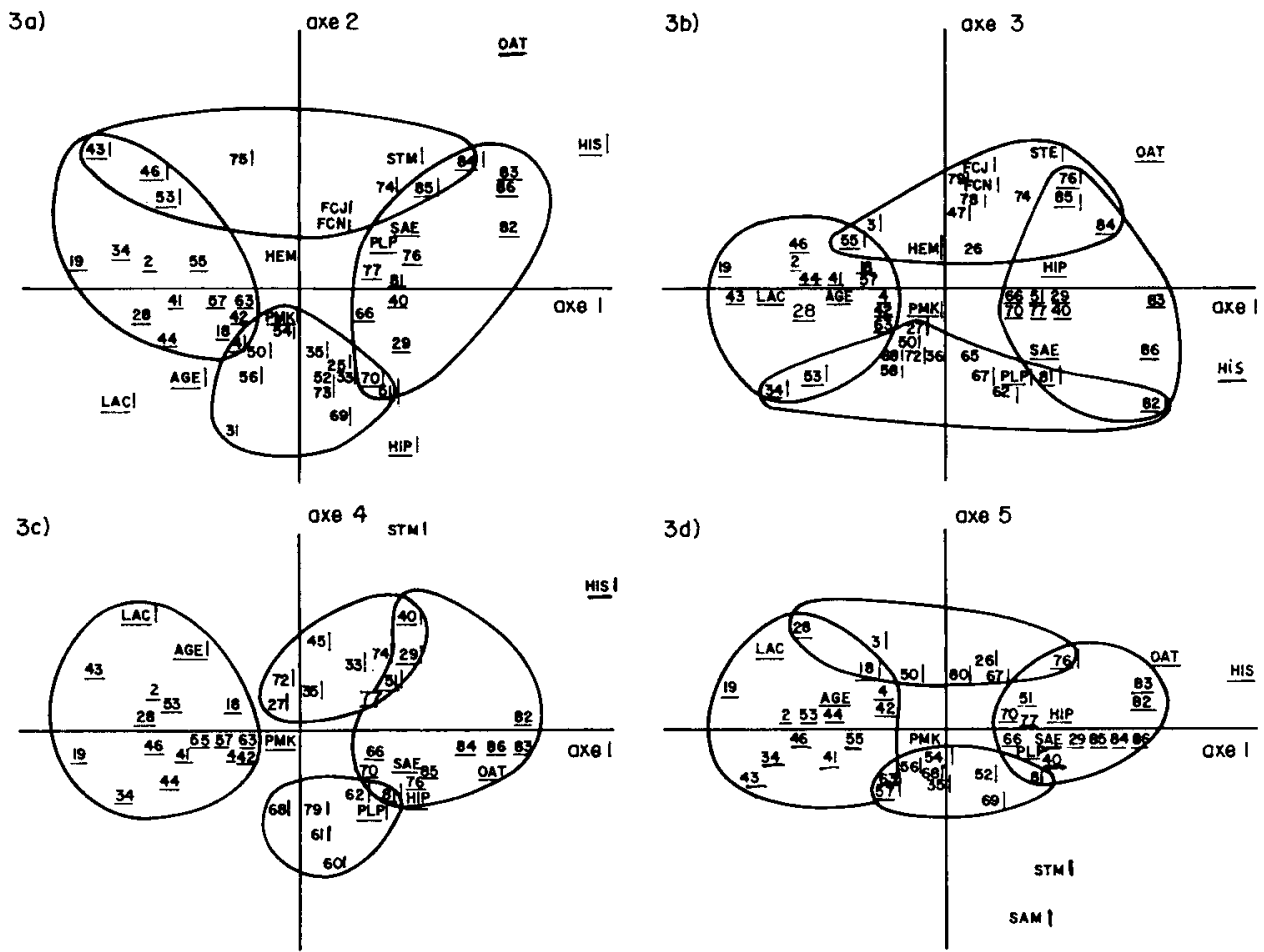

FIG. 3. - Représentation graphique de l'analyse de correspondance. Diagram of correspondance analysis.

- Les $\mathrm{n}^{\circ \mathrm{s}}$ représentent les vaches (the numbers corvespond to the cows).

- La signification des sigles des différents paramètres est rapportée à la légende du tableau I (the meaning of the sigla of the different parameters is given in the legend of table $I$ ).

- Les paramètres ou les vaches accompagnées d'un trait horizontal ou vertical sont représentés respectivement sur l'axe des abscisses et des ordonnées (parameters or cows accompanied by a horizontal or vertical line are represented on the axis of the abscissa or ordinate, respectively).

I. Etude du plan $x-2$ (fig. 3a).

a) Axe $T$.

Le tableau 3 indique que 9 paramètres sont représentés sur l'axe I.

Les 28 animaux représentés sur celui-ci se répartissent en 2 groupes distincts :

- le premier composé des nos $2,4, \mathbf{I} 8, \mathrm{I} 9,28,34,4 \mathrm{I}, 42,43,44,46,53,55$, 57 et 63 se situe près de l'index de dominance (HID). Si l'on sait que les proximités entre sujets sont proportionnelles à leur ressemblance et qu'ils sont caractérisés par les critères qui leur sont proches on peut donc dire que ces vaches seraient dominantes mais également :

- âgées et lourdes : proximité de LAC, AGE et PMK

- anciennes dans le troupeau : éloignement de OAT

- le second ( $\left.{ }^{\text {os }} 29,40,5 \mathrm{I}, 66,70,76,77,8 \mathrm{I}, 82,83,84,85,86\right)$ serait à l'inverse 
TABLEAU 3

Coefficients des contributions relatives des 20 paramètres sur les 5 axes des correspondances Coefficients of the velative contributions of the 20 parameters on the 5 axes of correspondences

\begin{tabular}{|c|c|c|c|c|c|}
\hline $\begin{array}{c}\text { Paramètres } \\
\text { (Parameters) }\end{array}$ & $\begin{array}{c}\text { Axe I } \\
(\text { Axis } T)\end{array}$ & $\begin{array}{c}\text { Axe } 2 \\
\left(\begin{array}{ll}\text { Axis } & 2\end{array}\right)\end{array}$ & $\begin{array}{c}\text { Axe } 3 \\
(\text { Axis } 3)\end{array}$ & $\begin{array}{c}\text { Axe } 4 \\
(\text { Axis } 4)\end{array}$ & $\left.\begin{array}{cc}\text { Axe } & 5 \\
(\text { Axis } & 5\end{array}\right)$ \\
\hline STM & 一 & I 2 & 一 & 35 & I 6 \\
\hline STE & - & - & I 2 & - & $\cdots$ \\
\hline SAM & - & - & - & - & 49 \\
\hline $\mathrm{SAE}$ & I 4 & 一 & - & $\ldots$ & - \\
\hline HID & 82 & I 6 & - & - & $\ldots$ \\
\hline HIS & 61 & $I_{4}$ & - & 15 & $\ldots$ \\
\hline HIP & 24 & $5^{8}$ & 一 & - & - \\
\hline PLE & 一 & 一 & 一 & - & 64 \\
\hline PLP & 27 & - & 29 & 23 & - \\
\hline ANR & 20 & 26 & - & - & - \\
\hline PMK & 28 & 25 & Io & - & - \\
\hline AGE & 29 & 23 & - & 17 & - \\
\hline $\mathrm{AGV}$ & - & 二 & 一 & -1 & - \\
\hline HEM & 一 & I 6 & Io & - & - \\
\hline LAC & 34 & I 5 & - & I 8 & $\ldots$ \\
\hline FCN & - & 22 & 58 & 一 & - \\
\hline$F C J$ & - & 20 & 64 & - & 一 \\
\hline$Q C J$ & 一 & $\ldots$ & - & 一 & - \\
\hline$\tilde{Q C N}$ & 一 & 一 & 一 & - & - \\
\hline QCE & 一 & 一 & 一 & 一 & $6 \mathrm{I}$ \\
\hline
\end{tabular}

composé de vaches dominées, légères, jeunes et récemment intégrées au chept 1 expérimental. Les coefficients de corrélation significatifs obtenus sur l'ensemble des 28 vaches entre les paramètres représentés sur l'axe I confirment les résultats obtenus par l'analyse des correspondances :

$$
\begin{array}{ll}
r /(\mathrm{HID} . \mathrm{AGE})=+0,8^{*} & r /(\mathrm{HIS} . \mathrm{AGE})=-0,67^{*} \\
r /(\mathrm{HID} . \mathrm{PMK})=+0,8^{*} & r /(\mathrm{HIS} . \mathrm{PMK})=-0,64^{*} \\
r /(\mathrm{HIS} . \mathrm{OAT})=+0,55^{*} & r /(\mathrm{HID} . \mathrm{OAT})=-0,55^{*}
\end{array}
$$

b) Axe 2.

20 vaches sont représentées sur l'axe 2 :

- I3 (n $\left.{ }^{\circ \mathrm{s}} 3,4,25,33,35,50,5 \mathrm{I}, 52,54,56,69,70,73\right)$ peuvent être considérées comme passives, lourdes, âgées (proches de HIP, PMK, LAC, AGE) et de condition physique médiocre puisque éloignées de HEM qui se trouve en opposition avec HIP sur l'axe 2,

- 7 dont la proximité de HID et de HIS mais aussi de HEM permet de les classer parmi les sujets actifs dominant (nos $43,46,53,75)$ ou actifs dominés (74, $84,85)$, en bonne condition physique.

Dans un tel cas également, les corrélations effectuées sur ces 20 vaches confirment les observations de l'analyse des correspondances :

$$
\begin{aligned}
& \mathrm{r} /(\mathrm{HIP} \cdot \mathrm{HEM})=-0,886^{*} \quad \mathrm{r} /(\mathrm{HIP} . \mathrm{AGE})=+0,42 \\
& \mathrm{r} /(\mathrm{HIP} \cdot \mathrm{PMK})=+0,09
\end{aligned}
$$


2. Etude du plan $I-3=$ axe $3($ fig. $3 \mathrm{~b})$.

Nous ne reviendrons plus sur l'axe I déjà étudié, le commentaire ci-dessous ne concerne que l'axe 3 . Les 23 sujets représentés sont également répartis en 2 groupes.

- Le premier a des coordonnées négatives et se situe près de PLP. Il comprend les vaches $n^{\text {os }} 27,34,36,5^{\circ}, 53,58,62,65,67,68,72,81,82$, qui sont donc de bonnes laitières. Compte tenu de leur proximité avec le poids (PMK) et de leur éloignement par rapport à la fréquence cardiaque (FCN, FCJ) et à l'écart de l'entrée en salle de traite (STE), nous pouvons conclure que ces animaux sont caractérisés par les critères suivants (en tenant compte de l'importance décroissante de leurs contributions relatives):

+ faibles fréquences cardiaques

+ fortes productions laitières

+ ordres d'entrée en salle de traite réguliers

+ poids élevés.

- Le second a des coordonnées positives et se situe près de FCX, FCJ. Il comprend les vaches $n^{\text {os }} 3,26,47,55,74,76,78,79,84,85$ qui ont de fortes fréquences cardiaques et, par opposition aux critères cités pour le I $^{\mathrm{er}}$ groupe, de faibles productions laitières, des ordres d'entrée en salle de traite irréguliers et des poids légers.

On peut donc retenir que les vaches qui rentrent d'une façon répétable en salle de traite sont les meilleures laitières $\left(^{(1)}\right.$ présentant une fréquence cardiaque basse (et inversement pour les mauvaises) :

$r /(\mathrm{PLP} . \mathrm{FCN})=-0,5 \mathrm{I}^{*}$; relations calculées sur l'ensemble des vaches

$r /($ PLP.STE $)=-0,2 \$$ représentées sur l'axe 3

\section{3. Étude du plan $I-7=$ axe 4 (fig. 3c).}

La distinction des 16 sujets représentés s'effectue là encore en 2 groupes distincts :

- Le premier a des coordonnées négatives et se situe autour de PIP. II comprend les vaches nos $60,6 \mathrm{I}, 62,68,79,8 \mathrm{I}$ qui sont de bonnes laitières; par opposition à STM, elles ont une faible médiane de l'ordre d'entrée ce qui signifit qu'elles accèdent dans les premières en salle de traite.

- Le second a des coordonnées positives et se situc autour de STM. Il comprend les vaches $\mathrm{n}^{\mathrm{os}} 27,29,33,35,40,5 \mathrm{I}, 72,74,77$ qui, inversement, d'une part, sont de mauvaises laitières et d'autre part, rentrent dans les dernières en salle de traite $(r /($ PLP.STM $)=-0,42)$.

4. Etude du plan $I-5=\operatorname{axc} .5$ (fig. $3 \mathrm{~d}$ ).

L'information contenue sur l'axe 5 étant de $5,6 \mathrm{p}$. Ioo nous n'attacherons: qu'une faible importance aux correspondances qui se révèleront sur celui-ci.

Nous obtenons aussi une répartition des 17 sujets en 2 groupes :

- Le premier a des coordonnées négatives et se situe près de SAM, SIM. Il est composé des vaches $n^{\text {os }} 35,52,54,56,57,63,68,69,8$ I caractérisées par leur entrée tardive en salle de traite et en salle d'attente, par une faible production laitière ainsi que par une faible quantité de concentré ingérée en salle de traite.

(1) Selon Incaex et Poutous (1965), le paramètre $\mathrm{PI}_{1} \mathrm{P}$ (quantité de lait maximum produite pendant 7 jours consécutifs en I re lactation) autait l'avantage de caractétiser le potentiel génétique dé chaque vache ct d'estimer précocement la production en 305 jours. 
- I.e second a des coordonnées positives. Il comprend les vaches $n^{0 \mathrm{~s}} 3$, I 8 , $26,28,50,67,76,80$ marquées par une entrée rapide en salle de traite (STM) et en salle d'attente (SAM), une bonne production laitière au cours de l'expérience (PLE) et une forte quantité de concentré ingérée en salle de traite (QCE). r/(PLE. $\mathrm{STM})=-0,70^{*} ; \quad r /(\mathrm{PLE} . \mathrm{SAM})=-0,74^{*}$.

\section{Rôle des paramètres $C P^{\prime} C$ et $C P^{\prime} G-\therefore$ choix des parcs d'attente.}

Notre analyse portait à l'origine sur 22 paramètres mais comme nous l'avons signalé précédemment, 2 d'entre eux ont dû être supprimés. En effet :

- l'information contenue par l'axe de cette première analyse était très grande (62 p. Ioo de l'information totale et 3 critères seulement y étaient représentés avec une contribution relative très forte pour CPG et CPC (respectivement 99 et $98 \mathrm{p}$. IOO) et plus faible pour OAT (I3 p. IOo). La quasi totalité des animaux se trouvaient donc subdivisés en 2 groupes sur les 4 plans autour de ces 2 paramètres très importants masquant ainsi l'intervention des 20 critères restants.

Nous pouvons toutefois tirer deux conclusions de cette première analyse: troupeau,

- le choix du parc d'attente est la caractéristique la plus importante de notre

- la proximité existant entre CPG et OAT signifie que les vaches qui choisissent le parc grillagé ont tendance à être les plus récemment arrivées dans le troupeair.

\section{C. - Résultats complímentaires à l'analyse des correspondances}

\section{Ordre d'entrée en salle de traite et ordre hiérarchique.}

Les coefficients de corrélation de Spearman obtenus après classement de I 2 vaches $\left({ }^{1}\right)$ en fonction de leur ordre d'entrée en salle de traite et de leur rang hiérarchique sont respectivement de - 0,03 et $-0,09$ selon que ce dernier est exprimé selon le (pouvoir) ${ }^{2}$ ou le pouvoir.

Ces valeurs non significatives permettent de conclure que pour les I 2 animaux que nous avons choisis, l'ordre d'entréc en salle de traite n'est pas déterminé par leur rang social ce qui confirme les résultats de l'analyse des correspondances.

\section{Relation entre l'ordre d'entrée en salle de traite et quelques stimuli provoquant l'éjection du lait.}

Après avoir étudié au cours de Io traites la fréquence d'apparition du réflexe d'éjection du lait consécutive à certaines stimulations d'origine mammaire (massage du pis, pose des gobelets, égouttage) ou extramammaire (entrée en salle de traite, distribution alimentairc), nous avons recherché s'il existait une corrélation entre la note ainsi attribuée à chaque animal (sur Io) et l'ordre d'entrée en salle de traite exprimé par le critère STM.

Les résultats sont présentés au tableau 4. Les deux coefficients négatifs significatifs entre STM et " entrée " d'une part $(-0,47)$ et STM et "concentré " d'autre part $(-0,80)$ traduisent le fait que les vaches qui rentrent les premières en salle de traite sont également celles pour lesquelles l'apparition du réflexe d'éjection est la plus fréquente lors de cette entrée et surtout lors de la distribution de concentré.

(1) Choisies pour la détermination plus précice du rangs social (voir matériel et méthodes). 


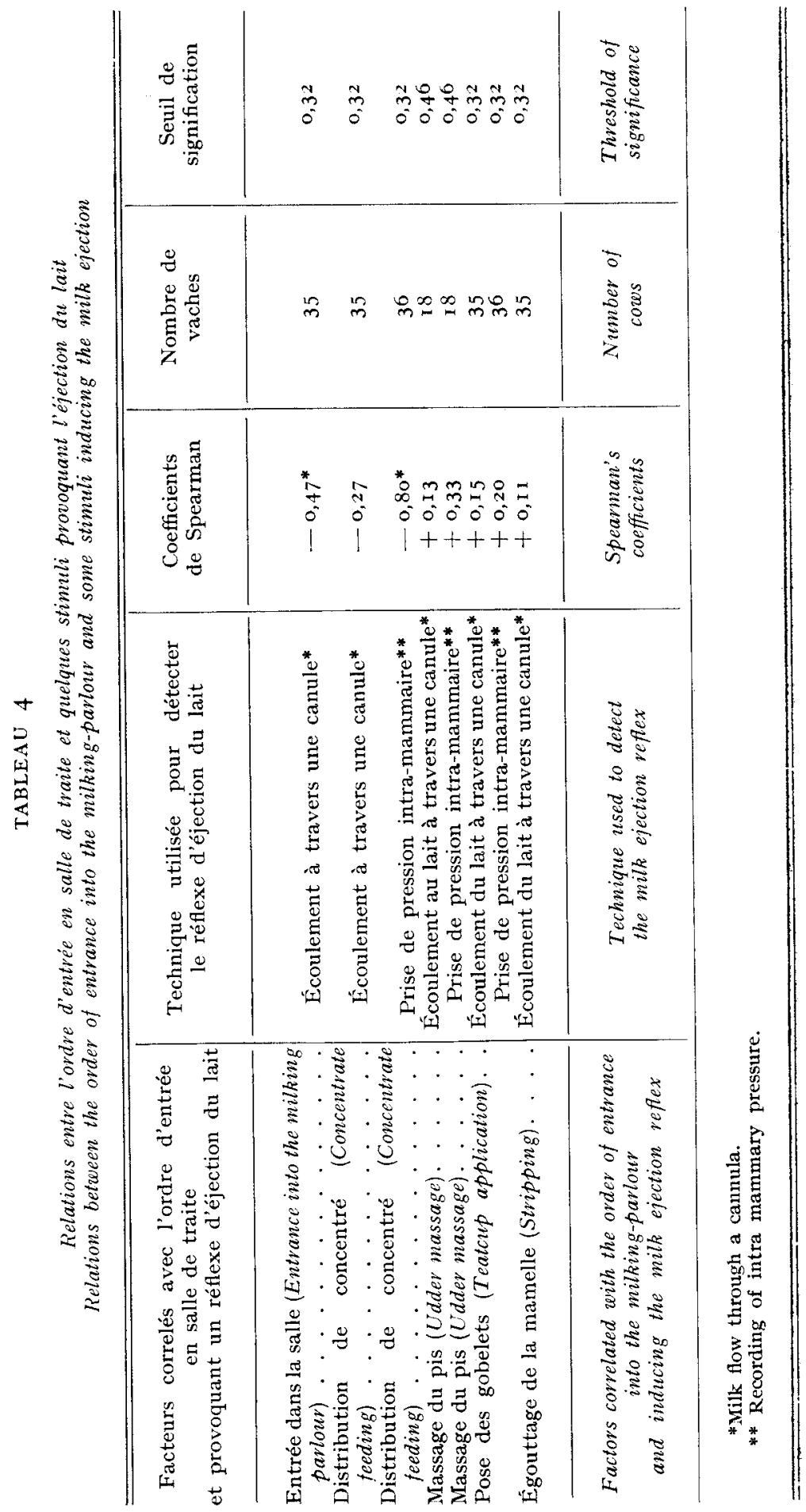


3. Effets de la distribution d'aliment concentré sur l'ordre d'entrée en salle de traite.

Après avoir observé l'ordre d'entrée en salle de traite d'un groupe de Io animaux recevant leur ration habituelle de concentré, nous avons totalement supprimé l'apport alimentaire sur les 5 vaches de tête alors que la quantité distribuée a été sensiblement augmentée sur les 5 vaches de queue. que :

L'expérience ayant été répétée une deuxième fois, pour confirmation, il ressort

-- Parmi les Io sujets (pour les 2 essais) qui ne reçoivent plus de concentré, tous sauf 2 retardent leur ordre d'entrée moyen avec un écart plus ou moins important $(+0, \mathbf{I}$ pour le no 28 et $+3,9$ pour le no 85$)$. C'est ainsi par exemple que la vache $n^{\circ}$ ig qui rentre $I^{\text {re }}$ au cours de toute la préexpérience commence à reculer son rang d'entrée à partir de la $6^{\mathrm{e}}$ traite et finit par rentrer $9^{\mathrm{e}}$ sur Io après avoir attendu plus de ro minutes dans le parc d'attente.

-- Parmi les ro sujets qui reçoivent la forte ration alimentaire, toutes sauf 3 avancent leur ordre d'entrée moyen dans des proportions variables (l'écart est de $-0,6$ pour le $n^{0} 57$ et de -- 5 , I pour le $n^{0} 79$ ) et la vache $n^{\circ} 89$ reste régulièrement la dernière.

\section{IV. - Discussion}

L'analyse des résultats obtenus au cours de ce travail nous conduit à faire les principaux commentaires suivants :

\section{I. - Les animaux choisissent leur parc d'attente avant de rentrer en salle de traite}

Bien qu'issue de conditions expérimentales légèrement différentes, cette conclusion est en accord avec celles de FERGusson et al., ig67 et de Hidari, Kido et SuzUki (I973).

S'il est difficile de préciser la motivation cxacte de ce choix, il faut souligner que, dans notre cas particulier, celui-ci est en rapport avec l'ancienneté des animaux dans le troupeau.

Les premiers sujets qui ont formé celui-ci sont généralement dominants; possédant ainsi la priorité de passage sur les vaches récemment arrivées ils auraient tendance à délaisser le parc grillagé où ils ont subi des manipulations apparemment mal supportées ${ }^{(1)}$.

\section{2. - L'ordre d'entrie en salle de traite est régulier}

Cette stabilité est admise par tous les auteurs (GUHL et ATKEson, I959; Willims et Lampo, I964; Dickson, Barr et Wieckert, ig67; Hidari, Kido et SUZL KI, I973; REINHARDT, I973) et bien que nous ne possédions pas de précisions: sur les travaux de certains d'entre cux (DieTrich et al., I965; FERGussox et al., I 967), on peut considérer ce fait comme bien établi compte tenu de la diversité des conditions expérimentales et des méthodes statistiques utilisées.

(1) P'iqûres, cathétérisation, canulation du trayon, etc. 
Par contre, nous avons pu montrer que l'accès en salle d'attente est l'objet de plus grandes variations. Ceci a également été signalé par Hidari, Kido et Suzuki, I973 et résulte peut-être de la perturbation occasionnée par la présence de l'ouvrier chargé de diriger les vaches vers les parcs d'attente.

\section{3. - Un groupe de bonnes laitières manifeste l'ordre d'entrie le plus stable et la fríquence cardiaque la plus basse (et inversement)}

Kostov et SubEv avaient déjà constaté en I970 que les animaux à faible production ne rentrent pas de façon régulière en salle de traite. L'axe 3 de l'analyse des correspondances nous a permis de mettre en évidence des faits analogues. I.a stabilité la plus importante est observée chez les bonnes laitières; malgré l'imperfection de la technique que nous avons utilisée, celles-ci se caractérisent par une basse fréquence cardiaque; ceci ne semble pas surprenant si l'on sait qu'un rythme lent se rencontre essentiellement chez des animaux en bon équilibre vago-synipathique pouvant se traduire par une faible émotivité qui les rend peu sensibles aux perturbations résultant des multiples modifications du milieu extérieur.

\section{4. -... Un groupe de bonnes laitières rentre en tête en salle de traite (et inversement)}

Ce résultat confirme les travaux de Millens et Lampo, ig64; Fergusson et al., I 967 ; Kostov et Subev, I970 et infirme ceux de Dietrich, I962; Dietrich et al., ig65; Hidari, Kido et Suzuki, i973; Reixhardi, I973.

La méthode des correspondances que nous avons utilisée permet peut-être d'expliquer cette divergence de résultats. Contrairement à un simple calcul de corrélations qui analyse globalement tous les animaux d'un troupeau pour trouver un lien entre 2 critères, l'analyse factorielle (qui n'est pas vraiment quantitative mais qualitative) présente l'avantage de dissocier les sujets en plusieurs groupes caractérisés par les mêmes facteurs. Elle met ainsi en évidence différents types d'animaux et l'on conçoit que le résultat du calcul des corrélations généralement utilisé par les auteurs, dépende en fait de l'existence et de l'importance numérique de ces différents types d'animaux au sein d'un troupeau.

\section{5. - Il n'y a pas de relation entre l'ordre d'entrée en salle de traite et l'hématocrite}

Il ressort des correspondances qui sont apparues sur l'axe 2 que les vaches actives ont un fort hématocrite (ct inversement) mais aucune relation ne s'est dégagéc entre celui-ci et l'ordre d'entrée. Il y a lieu do souligner qu'il faut se garder d'assimiler activité et dominance puisque Bocissoc (rg64) insiste sur le fait que l'animal dominant n'est pas forcément celui qui donne le plus de coups et que Hovels (I972) considère l'agressivité des sujets dominés comme la traduction de leur "frustration sociale". 


\section{6. -- L'ordre d'entrée en salle de traite n'est relié ni à l'âge ni au poids}

En effet, aucune correspondance entre ces critères n'est apparue au cours: de l'analyse factorielle et aucune corrélation n'est significative pour les 57 animaux du troupeau entre l'ordre d'entrée et l'âge $(-0,08)$ ou l'ordre d'entrée et le poids. $(+\mathrm{O}, \mathrm{OI})$.

Si tous les avis sont unanimes en ce qui concerne le rôle du poids (DIETRICH, ig62; Dietrich et al., ig65; Hidari, Kido et Suzlki, i973; Reinhardt, i973) il ne semble pas en être de même pour l'âge puisque Willems et LAMPo (I964); FERGUSSON et al. ( $\left.{ }^{96} 67\right)$ montrent que ce sont les animaux les plus âgés qui auraient tendance à rentrer en tête en salle de traite alors que pour KosTov et SLBEv (I970) cette particularité concerneraient les sujets qui ont seulement 5 à 6 ans.

\section{7. - Il n'y a pas de relation entre l'ordre hierarchique et l'ordre d'entrie en salle de traite}

En effet, aucune correspondance entre ces 2 critères n'est apparuc sur les axes et nous n'avons par ailleurs trouvé aucune corrélation entre l'ordre d'entrée ct l'ordre social $(-0,03)$.

Nous sommes en accord à cet égard avec les travaux de DiETRIch, I962, et ceux plus récents de DIC KSON, BARR et WIEC KERT (I967) qui pour leur part aboutissent à des relations du même ordre. Par contre, GuHz et ATkeson (I959) et REINHARDT (I973) citent des corrélations significatives respectivement de +0.52 et $+0,4 \mathrm{I}$.

Nous resterons toutefois réservés sur les conclusions de REiniariot qui classe ses 26 animaux selon un ordre social linéaire car les hiérarchies de ce type sont rares chez les mammifères (Bovissor et Sigxoret, I970). ReishardT souligne lui-même cet inconvénient et conclut comme Hidari, Kido et Suzuki (I973), que l'ordre social influence seulement l'ordre d'entrée en jouant un rôle modulateur : les vaches dominantes pourraient se servir de leur supériorité hiérarchique pour régler à leur profit les conflits sociaux à l'entrée en salle de traite et gagner ainsi quelques placess si elles le désirent (ce que nous avons nous-mêmes remarqué).

Si l'ordre social n'intervient pas dans l'ordre d'entrée en salle de traite il est possible à l'opposé cjue les liens, affectifs entre les animaux puissent jouer à cet égard un rôle non négligeable.

En effet, pour Hovels (1972), les bovins semblent rechercher la proximité de leurs compagnons d'élevage au pâturage, en stabulation et au râtelier; de plus, il existerait une faible agressivité à l'intérieur d'un groupe d'animaux ćlevés ensemble. Selon Leyhausex ( $97 \mathrm{r}$ ), il semblerait s'établir entre certaines vaches des rapports basés sur une sorte d'attraction personnelle que cet auteur assimile. à une forme d'amitié; celle-ci interviendrait dans toutes les activités du troupeau (marche, alimentation, repos, etc.) mais la composition des groupes d'attraction serait variable en fonction de chaque type d'activité.

\section{8. -. L'apport alimentaire en salle de traite peut avoir une infuence favorable sur l'ordre d'entrie.}

Les animaux qui se présentent les premiers en salle de traite sont apparemment ceux pour lesquels l'apparition du réflexe d'éjection du lait est la plus fréquente lors de l'apport alimentaire (corrélation significative de $+0,80$ ). 
Le rôle favorable de la distribution de concentré est confirmé :

- par nos propres essais d'augmentation ou de suppression de la ration qui provoquent respectivement une avance ou un recul significatifs de l'ordre de passage en salle de traite chez la plupart des sujets.

- par ceux de REINHARDT (I973) puisque sur II vaches recevant de la nourriture ad lıbitum avant l'heure habituelle de la traite, 9 d'entre elles sont rentrées plus tardivement.

Toutefois, il est important de remarquer que tous les animaux testés ne réagissent pas obligatoirement à ces modifications ( 15 sur 20 dans nos essais, 9 sur I I dans ceux de ReinhardT). D'ailleurs, Dietrich, I962 n'observe pas de changement significatif si une distribution de concentré précède ou suit l'accès en salle de traite.

Ces différentes réactions aux variations de ce facteur de l'environnement pourraient dépendre des facultés d'apprentissage des animaux qui sont très variables d'un individu à l'autre.

En effet, l'offre d'une récompense alimentaire est à la base de nombreux phénomènes d'apprentissage et de conditionnement.

C'est ainsi que pour AlBright et al. (I966), il faut 15 jours d'entraînement pour que $70 \mathrm{p}$. Ioo d'un troupeau de race Jersey apprenne à se succéder en salle de traite dans un ordre régulier au simple appel de leur numéro.

Ainsi, peut-on concevoir en se référant aux travaux de Gratchev (I952), Borsuk (i 955), Whittlestone (I956), Csiszar (I956), Dyusembix (I963), Zaks (Ig62), ETter (I964) et Brantas (I968b) que les stimuli qui précèdent l'éjection du lait (tels que le stationnement dans le parc d'attente, l'ouverture et le passage de la porte d'entrée en salle de traite, l'installation dans une stalle avec fermeture des barrières, le bruit des pulsateurs, l'entrée du vacher, l'apport alimentaire) sont susceptibles de devenir, selon les animaux, des signaux dont l'enchaînement peut être très complexe et qui sont continuellement renforcés par l'excitation. mécanique des trayons.

Dans ce contexte, et compte tenu de cette complexité les sujets qui, selon la terminologie Pavlovienne, sont de type "fort équilibrés mobiles ", pourraient être ceux qui rentrent les premiers en salle de traite. Dans un tel cas, les liaisons conditionnelles étant faciles à installer, stables et difficiles à inhiber, les animaux concernés "comprennent rapidement " qu'ils recevront au cours de la traite leur récompense alimentaire puisque celle-ci joue certainement un rôle essentiel dans le renforcement des enchaînements réflexes mis en cause.

Cette hypothèse qui mérite d'être vérifiéc, semble justifiée si l'on sait que pour KoKorina (I957), Barishnikov et Kokorina (I959 et I964) et Kudryavtzev ( I962) les vaches de ce type sont les meilleures laitières et qu'en ce qui nous concerne il se trouve que ce sont effectivement les vaches de tête qui produisent le plus de lait.

\section{C Conclusion}

A l'issue de cette premièrc étude, il nous apparaît possible de formuler quelques recommandations susceptibles d'améliorer l'organisation du travail sur le chantier de traite.

Le fait que les meilleures laitières accèdent généralement en tête dans la salle peut constituer un handicap dans les installations de type herring bone possédant un seul jeu de faisceaux trayeurs. Les éleveurs savent bien que la durée excessive de l'écoulement du lait de ces fortes productrices retarde non seulement la sortie 
de l'ensemble des vaches entrées en même temps qu'elles mais aussi le départ des séries suivantes. Celles-ci auraient pu en effet libérer les stalles plus rapidement s'il avait été possible de transférer plus tôt d'un quai à l'autre les gobelets immobilisés par les vaches longues à traire.

Dans ce contexte, il apparaît donc opportun :

Io D'équiper chaque moitié de fosse de son propre jeu de faisceaux trayeurs.

$2^{\circ}$ De constituer dans la stabulation des lots équilibrés en fonction du niveau de production afin de traire en dernier le groupe produisant le plus.

$3^{\circ}$ De proscrire l'emploi d'un "chien électrique " compte tenu des inhibitions de l'éjection du lait qu'il peut engendrer et de le remplacer par une barrière mobile qui est de loin préférable si l'on tient compte des facultés d'apprentissage des bovins.

La distribution du concentré au poste de traite semble à cet égard intéressante puisqu'il incite de nombreuses vaches (fortes, équilibrées) à venir se faire traire plus rapidement.

$4^{\circ}$ D'accroître la largeur des portes d'accès, voire de supprimer celles-ci en incorporant le parc d'attente à la salle de traite afin d'éviter que certains sujets dominants n'interdisent l'entrée aux vaches dominées.

Accepté pour publication en mars 1977.

\section{Summary}

\section{Psychophysiological determinism of dairy cow entrance into the milking parlour}

The order of entrance of 57 French Friesian Black and White cows and their choice between 2 "tandem " milking parlours and 2 adjoining waiting-pens (slatted and concrete floors) (fig. I) were checked 60 times at morning milking.

The following observations were made:

I) About 4 cows out of 5 (80.7 p. I oo choosed nearly always the same waiting-pen since 26.3 and 54.4 per cent of the herd went to the pen with slatted and concrete floor, respectively in more than 90 per cent of the cases (Table I).

$2^{\circ}$ The order of entrance into the milking-parlour was constant, but the access to the waiting-pen was more variable (Table 2, fig. 2).

The factorial analysis of correspondences (fig. 3) between the order of entrance of the animals and 22 parameters allowed to characterize the cows (Table I) and showed that:

I $^{\circ}$ A group of high yielding cows exhibited the most stable order of entrance and the lowest heart frequency. The correlation coefficient between maximum production in Ist lactation and heart frequency was -0.51 .

$2^{\circ}$ A group of high yielding cows entered first the milking parlour (and inversely).

$3^{\circ}$ There was no relationship between the hematocrit value and the order of entrance into the milking parlour.

$4^{\circ}$ The order of entrance was not related either to the age or to the weight of the animals.

$5^{\circ}$ The age and weight affected the hierarchical rank since dominant cows are often the oldest, the heaviest and the most ancient in the herd (and inversely).

$6^{\circ}$ The order of entrance into the milking parlour did not seem to depend on the hierarchical order, but some dominant cows might use their " superiority " to settle some quarrels to their advantage and thus gain a few places.

A study complementary to the analysis of correspondences showed that the cows arriving first in the milking parlour were also those exhibiting the most frequently the milk ejection reflex when entering the parlour and especially when receiving the feed at the milking unit (the correlation between these 2 stimuli of milk ejection and the entrance order were -0.47 and 0.80 , respectively; (Table 4).

Supply of concentrate in the milking parlour seemed to facilitate the access of the animals to this area and suppression of the ration delayed the order of entrance in 80 per cent of the ani- 
mals. It may be assumed that the stimuli preceding the milk ejection such as standing in the waiting-pen, opening of the door and entrance into the milking parlour, installation in a box, noise of pulsators, arrival of the milker, allowance of feed, might become signals the very complex sequence of which might be continuously enhanced by the mechanical stimulation of the teats. In this context, the subjects of the type "strong, balanced, mobile " of Pavlov's terminology might be those entering first the milking parlour.

\section{Références bibliographiques}

Albright J. L., Gordon W. P., Black W. C., Dietrich J. P., Snyder W. W., ig66. Behavioral responses of cows to auditory training. J. Dairy Sci., 49 (I), 104-106.

BARISHNi KOV I. A., KoKorXNA E. P., 1959. Higher nervous activity and lactation. I $5^{\text {th }}$ internat. dairy Congr. London, 1959, 1, 46-53.

Barishni kov I. A., Kokorina E. P., 1964. Higher nervous activity of cattle. Dairy Sci. Abstr., 26 (3), 97-I 5.

Borsuk V. N., 1955. Conditionned reflex regulation of milk removal from the ducts of the udder in cows. 2nd Conference on the physiology of farm animals, summary of proceedings, Moscow, Leningrad.

Bourssou M. F., 1964. Observations sur la hiérarchie sociale chez les bovins domestiques. D.E.S., Fac. Sci., Paris.

Bousssou M. F., I97o. Technique de mise en évidence des relations hiérarchiques dans un groupe de bovins domestiques. Rev. Comp. Animal, 66-69.

Bouissou M. F., Signoret J. P., I970. La hiérarchie sociale chez les mammifères. Rev. Comp. Animal, 43-6r.

Brantas G. C., I968b. Training, eliminative behaviour and resting behaviour of Friesian. Dutch cows in the cafetaria stable, Zeit. Tievzucht. Zucht-Biol., 85 (I), 64-77.

Csiszar V., I956. Der Viilcheinschuss als Redingter Reflex und die Arten der Hemmungen. I $4^{\text {th }}$ internat. Dairy Congr., Roma, 1, I1 7-125.

Decaen C., Poutous M., I965. Phase ascendante de la courbe de lactation chez la vache laitière. Ann. Zootech., 14 I 35-I43.

Dickson D. P., Barr G. R., Wieckert D. A., i 967 . Social relationship of dairy cows in a feed. lot. Behaviour, 29, I95-203.

Dietrich J. P., 1962. Behavioral patterns in dairy cattle as affected by management. Dissert. Abstr., 23, 769-770.

Dietrich J. P., Snyder W. W., Meadows C. E., Albright J. L., I965. Rank order in dairy cows. Amer. Zoologist., 5, 713.

Dyusembin Kh., I963. Milking without suckling. Dairy Sci. Abstr., 27, r666.

ETter E. W., 1964. Means and method for tending domestic animals. Off. Gaz. U.S. Patent Office, $803(3), 719-72 \mathrm{I}$.

Fergusson R. S., Albright J. L., Harrington R. B., Black W. C., Donaldson S. L., Snyder W. W., Dietrich J. P., I967. Dairy cattle entrance behavior into a milking area. Amer. Zoologist., 7, $807-808$.

Gratchev I. I., I952. Formation du réflexc conditionné de sécrétion lactée basé sur l'excitation mécanique d'un quartier. Dokl. Akad. Nauk., SSSR, 86, 441-444 (en russe).

Guhl A. M., At Keson F. W., 1959. Social organization in a herd of dairy cows. Trans. Kansas Acad. Sci., 62, 80-87.

Hidari H., Krdo M., Suzuki S., 1973. The order of entry of cows into a milking parlour under loose-housing conditions. Jap. J. Zootechn. Sci., 44, 33-38.

Hovels J., I 972 . Observations sur le comportement social des bovins domestiques. Effet des conditions d'élevage. Mémoire d'étude. Labo. Études du comportement 37380 Nouzilly.

Kokorina E. P., 1957. Détermination des types d'activité nerveuse supérieure en liaison avec la production de lait. Vopr. Fiziol. Sel "skokoz. Zivot, 44-49.

Kostov S., Subev S., 1970. Behaviour of cows under loose housing conditions and during milking in a herringbone parlour. I. Order of entering parlour. Dairy Sci. Abstr., 32, 404.

KudRyAvtzev A. A., 1962. Higher nervous activity and the physiology of the senses in lactating cows. I6th internat. Dairy Congr., Copenhague, 1962, 565-572.

LABUSSiÈre J., I975. Limitations des servitudes de la traite. 1'e partie : choix des méthodes et des équipements. In: Journées d'études de l'I.T.E.B. Paris, $\mathrm{r}_{4}$ et I 5 janvier 1975.

Labussik̀re J., Durand A., 1970. La pression intramammaire chez les bovins après une stimulation de la mamelle ou une injection intrajugulaire d'ocytocine. Ann. Zootech., 19, $3^{8.5-}$ 397 . 
Leyhausen P., I97I. Behavior and environment. New York, Iondon: Plenum Press.

REINHARDT U. V., r973. Reitrage zur sozialen Rangordnung und Melk ordnung bei Kühen. Z. Tierpsychol., 32, $281-292$.

Whittlestone W. G., 1956. The fundamentals of efficient milking. I 4 th internat. Dairy Congr., Roma, 1, 356-368.

Willems A. E. R., LAmpo P., 1964. De rangorde bii het melken van Koeien in de meldstal. Vlaams diergeneekd. T., 33 (3), 75-82.

ZAKs M. G., 1962. The motor apparatus of the mammary gland. Edinburgh : Cowie, Oliver and Boyd, 86-104, London. 